\title{
Prevalence of Cervical Cancer and Associated Risk Factors among Women Attending Cervical Cancer Screening and Diagnosis Center at Yirgalem General Hospital, Southern Ethiopia
}

\section{Tesfahun Hailemariam ${ }^{1 *}$, Bizunesh Yohannes ${ }^{2}$, Hiwot Aschenaki ${ }^{3}$, Engidawork Mamaye ${ }^{4}$ Goye Orkaido $^{5}$ and Mihret Seta ${ }^{6}$}

${ }^{1}$ Department of Health Informatics, Hawassa College of Health Science, Hawassa, Ethiopia

${ }^{2}$ Bonbe Primary Hospital, SNNPR, Ethiopia

${ }^{3}$ Derashe Primary Hospital, SNNPR, Ethiopia

${ }^{4}$ Omorate Health Center, SNNPR, Ethiopia

${ }^{5}$ Arba Minch College of Health Science, SNNPR, Ethiopia

${ }^{6}$ Wolita Sodo Teaching and Referral Hospital, SNNPR, Ethiopia

\begin{abstract}
Introduction: A worldwide estimated annual case of cervical cancer is 493,000 and ends with 273,500 annual deaths. In developing countries, it accounts for about $85 \%$ of both its morbidity and mortality. According to the report from World health organization, globally in 2012 , cervical cancer incidence was $7.9 \%$, mortality $7.5 \%$ and five-year prevalence was $9 \%$. In sub-Saharan Africa the incidence was $25.2 \%$, mortality $23.2 \%$ and five-year prevalence was $27.6 \%$. In Ethiopia the incidence was $17.3 \%$, mortality $16.5 \%$ and five-year prevalence was $18.2 \%$.
\end{abstract}

Methods: Retrospective cross-sectional study was conducted between 10/08/08 up to 28/01/2009 E.C. Data was collected from August $17^{\text {th }}, 2002 \mathrm{E} . \mathrm{C}$ to $2008 \mathrm{E}$.C all charts of cervical cancer patients diagnosed and screened between August $17^{\text {th }}$, 2002 E.C to 2008 E.C at Yirgalem General Hospital was retrieved from cervical cancer screening and diagnosis logbook. Univariate, bivariate and multivariate analysis were performed using SPSS version 20 .

Results: from the screened clients, $16.5 \%$ had cervical cancer. From the screened and diagnosed clients those with multiple sexual partners had 40 times higher odd of cervical than those with no multiple sexual partners. This study revealed that being Human immune deficiency virus positive (AOR=9.033: 95\%: $\mathrm{Cl} 4.537,17.985$ ), sexually transmitted infection history (AOR=8.364:95\% Cl: $5.639,12.405)$ and early age at initiation of sexual intercourse (AOR=8.968:95\%: Cl 5.588, 14.393) have statistically significantly associated with cervical cancer in multivariate analysis

Conclusion: Of screened clients, $16.5 \%$ were with acetone white lesion and the risk factors for cervical cancer were having multiple sexual partners, human immune deficiency virus positive, history of sexually transmitted infection and early age at initiation of sexual intercourse. Early screening on mass campaign and focus from the government and other stake holders by strengthening both cancer prevention and control program and implementation strategies through due attention on the associated risk factors of the study.

Keywords: Cervical cancer; risk factors; Prevalence multivariate analysis

Abbreviations: CC: Cervical Cancer; CCS: Cervical Cancer Screening; CIN: Cervical Intraepithelial Neoplasia; HSIL: High Grade Squamous Intraepithelial Lesion; HPV: Human Papilloma Virus; HR HPV: High Risk Human Papilloma Virus; SIL: Squamous cell Intraepithelial Lesion; SNNPR: Southern Nations Nationalities and People's Region; VIA: Visual Inspection of Acetic Acid; MSP: Multiple Sexual Intercourse; YGH: Yirgalem General Hospital

\section{Introduction}

Cervical cancer is a cancer arising from the cervix, in which the cells of the cervix become abnormal and start to grow uncontrollably, forming tumor. Approximately $90 \%$ of intraepithelial neoplasia is attributed to human papillomavirus (HPV) infection. Only certain types of HPV cause high grade intraepithelial lesions and cancer. The most common HPV are HPV-16, -18, -31, -33, -35, -39, -45, -51, -52, -56, and -58. Type 16 is the most common form of HPV found in invasive cancer and in CIN 2 and CIN 3 and HPV-18 is more specific than HPV-16 for invasive tumors- found lower (2\%) in negative finding. In most women, the infection will clear in 9 to 15 months. Any factor that influences the integration of HPV DNA into the human genome may cause progression to invasive disease. The two major histologic types of cervical cancer are adenocarcinoma and squamous cell carcinoma. From this $99 \%$ of women with squamous cervical carcinoma caused by Human Papilloma virus (HPV) infection [1].

The risk for developing cervical cancer is associated with early age of first intercourse, multiple sexual partners, smoking and infection with $\mathrm{HPV}$, use oral contraceptive for a longer period and occupation are the risk factors for the developing cervical cancer [1]. In addition, a woman who has sexual intercourse with a male partner who in turn has had

*Corresponding author: Tesfahun Hailemariam, Department of Health Informatics, Hawassa College of Health Science, Hawassa, Ethiopia, Tel: 251934107979; E-mail: tesfahunhailemariam@gmail.com

Received October 22, 2017; Accepted November 27, 2017; Published November 30, 2017

Citation: Hailemariam T, Yohannes B, Aschenaki H, Mamaye E, Orkaido G, et al. (2017) Prevalence of Cervical Cancer and Associated Risk Factors among Women Attending Cervical Cancer Screening and Diagnosis Center at Yirgalem Genera Hospital, Southern Ethiopia. J Cancer Sci Ther 9: 730-735. doi:10.4172/19485956.1000500

Copyright: (C) 2017 Hailemariam T, et al. This is an open-access article distributed under the terms of the Creative Commons Attribution License, which permits unrestricted use, distribution, and reproduction in any medium, provided the original author and source are credited. 
intercourse with multiple women also confers as a significant risk to develop cervical cancer [2,3]. Majority of the cases are associated with an increased risk of acquiring or having inappropriate compromised immune response to infection with HPV, the etiologic agent of most cervical cancer [1].

A non-communicable disease like cervical cancer creates devastating effects in the developing countries. According to the World Health Organization (WHO) and international union against cancer, 24.6 million people are living with cancer around the world. In 2002 and 2008, cancer accounted for the deaths of 7.6 million people [1,4]. Globally cancer is the fifth and the second most frequent malignancy in men and women respectively. Overall, 715,000 new cancer cases and 542,000 cancer deaths were estimated occurrence in Africa [4,5].

Approximately half a million women develop cervical cancer each year with an estimated $85 \%$ in developing countries [4,5]. It is the most common among women of sub-Saharan Africa following breast cancer in northern Africa [6]. In South Africa, cervical cancer is the most common cancer in black women and fourth among white women. Among women in developing countries those who have appeared with advanced disease, it remains a major cause of morbidity and mortality [7].

A considerable reduction in cervical cancer (CC) incidence and deaths has been achieved in developed countries with systematic cytological screening programs. Thus, it is largely preventable by effective screening programs. However, this has not been possible in most limited resource countries mainly because of systematic screening is rarely performed [8]. In Ethiopia, Cervical Cancer ranks as the $2^{\text {nd }}$ most frequent cancer among women between 15 and 44 years of age. Every year, 4648 women are diagnosed with cervical cancer and of this, 3,235 ends with death [9].

Generally, worldwide trends show that developing countries going through rapid societal and economic changes. The shift towards the lifestyles of industrialized countries leads to a rising burden of cancers associated with reproductive, dietary, and hormonal risk factors [1033]. In 2013, WHO launched the Global Action Plan for the Prevention and Control of Non-Communicable Diseases from 2013-2030. The aim is to reduce premature mortality from Cancer, Cardiovascular diseases, Diabetes and Chronic respiratory diseases by $25 \%$.

In Ethiopia, despite there was no separate cervical cancer prevention strategy and screening program, Ethiopian reproductive health strategy gives due attention to reproductive organ as one of its components. There is also project named "Adistesefa" by pathfinder international in Ethiopia that supports comprehensive facility services, educate the community about cervical cancer prevention, and establish lasting alliances with local partners. Together with the Federal Ministry of Health (FMOH) and Stanford University Program for International Reproductive Education and Services (SPIRES), the project aims to establish services in 14 health institutions (including five Cervical Cancer Prevention Centers of Excellence) in the regions of Addis Ababa, Amhara, Oromia, Tigray, and Southern Nations Nationalities and People's Region (SNNPR) [10].

Recently, FMOH launched guide line for cervical cancer prevention which aims to provide healthcare providers, implementing partners and other stake holders involved in the prevention and control of cervical cancer in Ethiopia with standardized cervical cancer prevention and control health service delivery directive. And From the pilot project with path finder, FMOH planned to scale up screening service to public health care facilities.
Cervical cancer is the commonest cancer cause of death among women in developing countries. "Mortality due to cervical cancer is also an indicator of health inequities, as $85 \%$ of all deaths due to cervical cancer are in developing, low- and middle-income countries [1] According to the report from WHO, globally in 2015, cervical cancer incidence was $7.9 \%$, mortality was $7.5 \%$ and five-year prevalence was 9\%. In sub-Saharan Africa the incidence was $25.2 \%$ and mortality was $23.2 \%$ and the five-year prevalence was $27.6 \%$. In Ethiopia the incidence was $17.3 \%$ was mortality $16.5 \%$ and five-year prevalence was $18.2 \%$ [4]

Cervical cancer is the commonest cancer affecting reproductive organ and leading cause of death among women]. It is one of the registered Cancers with increasing incidence trends in the US: 19992008 [1]. Internationally, the burden of cervical cancer falls most heavily on developing nations. About $85 \%$ of the cases and $88 \%$ of the deaths due to cervical cancer occur in developing nations. Women in developing nations are at a 35\% greater lifetime risk of developing cervical cancer than women in high-income countries. Although cervical cancer is most common in women older than 50 years, in developing nations, it is becoming increasing prevalent among women during their reproductive age 15-49 years [6]

It was estimated that 20.9 million women were at risk of developing cervical cancer in Ethiopia with an estimated 4648 annual number of new cases and 3235 annual number of deaths respectively [5]. Cervical cancer is the second most common cancer and the fourth leading cause of Cancer related deaths among women worldwide. Currently Ethiopia has a population of 20.9 million women aged 15 and older who are at risk of developing cervical cancer. Each year an estimated 7619 women are diagnosed with cervical cancer; 6081 die from the disease. Crude incidence rates of cervical cancer in Ethiopian women per 100000 population per year are estimated to be 23 [1]. Early screening on mass campaign and focus from the government and other stake holders by strengthening both cancer prevention and control program and implementation strategies through due attention on the predictors of cervical cancer is vital. Therefore, the aim of this study is to investigate the prevalence and associated risk factors of cervical cancer among clients that have been screened for cervical cancer in Yirgalem general hospital.

Despites the growing number of cervical cancer cases in Ethiopia, still there is a gap in knowledge of risk factors. By considering the increasing pattern of the disease and high prevalence of risk factors, the need for cervical cancer prevention program is crucial. Data from the primary health care facilities are also scarce to see the problem for better intervention. To establish and improve any program and strategy, understanding the prevalence and associated risk factors of cervical cancer is become important. In Ethiopia Information related to cervical cancer like knowledge of the disease, attitude and practice towards screening is very limited. Identification of the factors associated with cervical cancer will help the Ministry of Health and Social Welfare in development of a cervical cancer health promotion and preventive strategy to mitigate the prevalence and the lethality of the case. Furthermore, it helps health specialists, policy makers, and planners on cervical cancer prevention; which is a practical method for a setup with no universal national HPV vaccine coverage like Ethiopia. In addition, the finding of this study will point in making evidence based public health actions and refine the preventive activities within the national cervical cancer preventive and control strategies. 
Citation: Hailemariam T, Yohannes B, Aschenaki H, Mamaye E, Orkaido G, et al. (2017) Prevalence of Cervical Cancer and Associated Risk Factors among Women Attending Cervical Cancer Screening and Diagnosis Center at Yirgalem General Hospital, Southern Ethiopia. J Cancer Sci Ther 9: 730-735. doi:10.4172/1948-5956.1000500

\section{Methods}

\section{Study setting and study design}

Yirgalem General Hospital is found in Sidaman zone, located in southern nations, nationalities and people's regional state (SNNPR). Sidama zone is one of the zones found in SNNPR; the zone has 19 woredas and four city administrations. Yirgalem town is one of the four city administrations andwhich is located at $317 \mathrm{~km}$ from Addis Ababa the capital city of Ethiopia and $47 \mathrm{~km}$ from Hawassa, the capital of SNNPR and Yirgalem general Hospital is found in Yirgalem town. It is the largest public hospital which was built in the early 1954 E.C with approximately 230 inpatients beds. The Yirgalem General Hospital Cervical Cancer Treatment Center was established seven years ago. Currently two nurses working at the Cervical Cancer Center. Retrospective cross-sectional study was conducted between 10/08/08 up to $28 / 01 / 2009$ E.C. Data was collected from August $17^{\text {th }}, 2002$ E.C to 2008 E.C all charts of cervical cancer patients diagnosed and screened between August 17 $7^{\text {th }}, 2002$ E.C to 2008 E.C at Yirgalem General Hospital was retrieved from cervical cancer screening and diagnosis logbook that met the inclusion criteria.

\section{Sample size determination and sampling procedure}

All women who have attended cervical cancer screening and diagnosis center at Yirgalem General Hospital. Non-probable purposive samplings were used. Data was collected from secondary data using checklist. And, the data collectors were those who work at the cervical cancer screening and treatment center.

\section{Data quality}

The checklists filled were gathered and checked for completeness. Extracted data was entered in to statistical package of social science software version 20 and univarate, bivarate and multivarate analysis were performed.

\section{Ethical issues}

The ethical issue was assured by Hawassa College of health science internal review board for ethical approval. Consent was obtained from administrative body and hospital staffs. And all data collected were kept confidentially and study was conducted over the dictated time.

\section{Results}

A total of 2120 patient with acetowhite lesion and no acetowhite lesion were registered on Yirgalem general hospital cervical cancer registration log book. During data collection, 1945 were included in the study and the rest 175 cases were completely excluded due to missing of one or more study variables. Out of the total extracted data, 1805 $(92.8 \%)$ were in the age $\geq 35$ years and mean age of the patients was $41.66 \pm 7.68 .1210(62.2 \%)$ of the clients were living in urban and the remaining 733 (37.8\%) were living in rural. Regarding marital status $1459(75 \%)$ were married and the rest 200 (10.3\%), 175 (9\%), 89 (4.6\%) were, divorced, widowed, and single respectively. The majority of the cases, 1534 (78.9\%) of the client's educational status was literate and the remaining $411(21.1 \%)$ were no able to read and write. 1106 $(59.6 \%)$ of clients were employed. and the remaining 839 (43.1\%) were unemployed (Table 1). Out of the study population, 321 (16.5\%) were detected as aceton white lesion positive and the remaining were negative for acetowhite lesion. Concerning the status of the study subjects' risk factors, some of reproductive and behavioral risk factors for cervical cancer were multipara 1360 (69.9\%), grand multipara, $484(24.9 \%)$ and nulipara 101 (5.2\%) respectively. According to the study, 1487 (76.5\%) were contraceptive users and the remaining 458 (23.5\%) were non-users. History of STI 468 (24.1\%) have STI history and the remaining have no STI history, HIV/AIDS 64 (3.3\%) were positive for HIV status and the remaining 1880 (96.7\%) were negative for HIV status, regarding to age at first intercourse about $1220(62.7 \%)$ were age $<18$, and the rests 725 (37.3\%) were age $>18$. Cigarette smoking about 95 (4.9\%) were smoking cigarette and the rests are non-smokers, and concerning to multiple sexual partners $210(10.8 \%)$ were have had multiple sexual partners and the remaining are not have had multiple sexual partners (Table 2).

\begin{tabular}{|c|c|c|}
\hline Socio-demographic variable & Frequency & Percent (\%) \\
\hline \multicolumn{3}{|c|}{ Age of the women } \\
\hline$<35$ & 140 & 7.2 \\
\hline$\geq 35$ & 1805 & 92.8 \\
\hline \multicolumn{3}{|c|}{ Address } \\
\hline Urban & 1210 & 62.2 \\
\hline Rural & 735 & 37.8 \\
\hline \multicolumn{3}{|c|}{ Marital status } \\
\hline Single & 89 & 4.6 \\
\hline Married & 1459 & 75.0 \\
\hline Divorced & 200 & 10.3 \\
\hline Widowed & 175 & 9.0 \\
\hline Others & 22 & 1.1 \\
\hline \multicolumn{3}{|c|}{ Occupation } \\
\hline Employed & 1106 & 56.9 \\
\hline Unemployed & 839 & 43.1 \\
\hline \multicolumn{3}{|c|}{ Educational status } \\
\hline Illiterate & 411 & 21.1 \\
\hline Literate & 1534 & 78.9 \\
\hline
\end{tabular}

Table 1: Socio-demographic characteristics of women attending oncology center at Yirgalem General Hospital in the period between 2002 E.C-2008 E.C.

\begin{tabular}{|c|c|c|}
\hline Associated factors for CC & Frequency & Percentile (\%) \\
\hline \multicolumn{3}{|c|}{ Parity } \\
\hline 0 & 101 & 5.2 \\
\hline $1-5$ & 1245 & 64.0 \\
\hline$\geq 5$ & 593 & 30.5 \\
\hline \multicolumn{3}{|c|}{ Use of contraceptive } \\
\hline Yes & 1487 & 76.5 \\
\hline No & 458 & 23.5 \\
\hline \multicolumn{3}{|c|}{ History of STI } \\
\hline Yes & 468 & 24.1 \\
\hline No & 1477 & 75.9 \\
\hline \multicolumn{3}{|l|}{ HIV status } \\
\hline Negative & 1880 & 96.7 \\
\hline Positive & 65 & 3.3 \\
\hline \multicolumn{3}{|c|}{ Age at first intercourse } \\
\hline$<18$ & 1220 & 62.7 \\
\hline$\geq 18$ & 725 & 37.3 \\
\hline \multicolumn{3}{|c|}{ Cigarette smoking } \\
\hline Yes & 95 & 4.9 \\
\hline No & 1850 & 95.1 \\
\hline \multicolumn{3}{|c|}{ Multiple sexual partner } \\
\hline Yes & 210 & 10.8 \\
\hline No & 1735 & 89.2 \\
\hline \multicolumn{3}{|c|}{ VIA } \\
\hline Yes & 321 & 16.5 \\
\hline No & 1624 & 83.5 \\
\hline
\end{tabular}

Table 2: Reproductive and behavioral characteristics of study population Yiregalem, Ethiopia, and $17^{\text {th }}$ August 2002 E.C-2008 E.C 
Citation: Hailemariam T, Yohannes B, Aschenaki H, Mamaye E, Orkaido G, et al. (2017) Prevalence of Cervical Cancer and Associated Risk Factors among Women Attending Cervical Cancer Screening and Diagnosis Center at Yirgalem General Hospital, Southern Ethiopia. J Cancer Sci Ther 9: 730-735. doi:10.4172/1948-5956.1000500

Out of the total clients, 321 (16.5\%) were acetowhite lesion positive and the remaining 1624 (83.5\%) were negative for acetowhite lesion (Figure 1). The majority 125 were diagnosed in 2003 and the rest $3,67,90,7$, 17,12 were diagnosed in 2002, 2004,2005,2006,2007 respectively. 140 (43.75\%) women with acetowhite lesion were manifested with post coital bleeding (Figure 2). The likelihood of having cervical cancer among urban residents was 2.766 times higher as compared to those living in rural (COR=2.766: 95\% CI: 2.071,3.695). The likelihood of getting cervical cancer among women who reported as marital status single was significantly associated as compared to married women (COR=5.4: 95\% CI: 3.489,8.387) and the likelihood of cervical cancer among literate women was 6.1 times higher as compared to with those illiterate women (COR=6.1: 95\% CI: $6.13 .660,10.257)$. According to the study, employed women were 3.586 times more likely to have cervical cancer as compared to unemployed women (COR=3.586: $95 \%$ CI: 2.686, 4.789) (Table 3).

This study shows that, multipara women were 3.484 times more likely to have cervical cancer as compared to nuilipara women (COR=3.484: 95\% CI: 2.289, 5.304). The odds of cervical cancer among ever users of oral contraceptive were significantly increased by $97 \%$ as compared to never users of oral contraceptives (COR=1.975: $95 \%$ CI:1.421, 2.745). Age at first sexual intercourse women's age less than 18 was 3.756 times risk to develop cervical cancer than those age $>18$

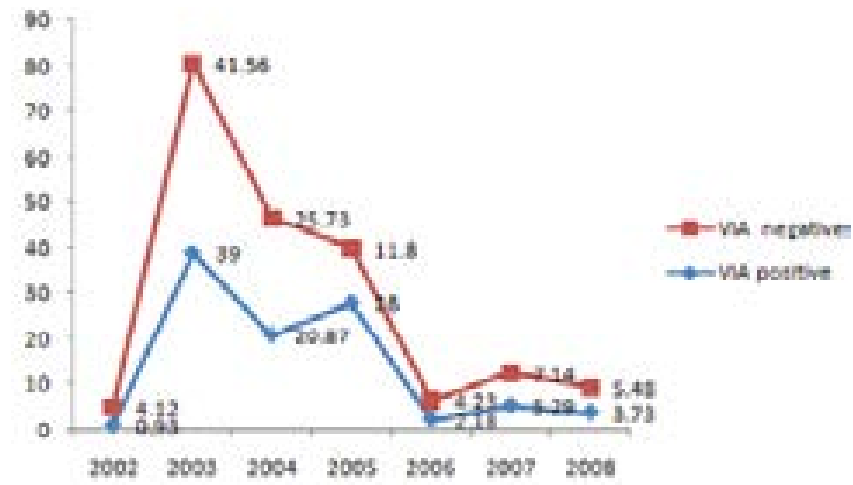

Figure 1: Trend of cervical cancer over five years.

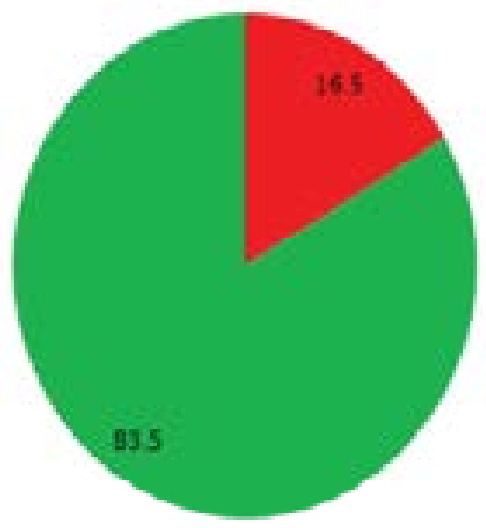

Figure 2: Prevalence of cervical cancer at the study area.

\begin{tabular}{|c|c|c|c|c|c|}
\hline \multicolumn{2}{|c|}{ Variables } & VIA positive & VIA negative & COR for $95 \% \mathrm{Cl}$ & AOR for $95 \% \mathrm{Cl}$ \\
\hline \multirow{2}{*}{ Age } & $<35$ & 43 & 97 & 1 & - \\
\hline & $\geq 35$ & 278 & 1527 & $0.4(0.2810 .601)$ & ** \\
\hline \multirow{2}{*}{ Address } & Urban & 256 & 65 & $2.7(2.071-3.695$ & ** \\
\hline & Rural & 954 & 670 & 1 & - \\
\hline \multirow{4}{*}{ Marital status } & Single & 45 & 44 & $5.4(3.4898 .387)$ & ** \\
\hline & Married & 232 & 1227 & 1 & - \\
\hline & Divorced & 39 & 161 & $1.2(8791.868)$ & ** \\
\hline & Other & 5 & 192 & $0.2(0.034 .881)$ & ** \\
\hline \multirow{2}{*}{ Occupation } & Employed & 256 & 850 & $3.5(2.686-4.78)$ & ** \\
\hline & Unemployed & 65 & 774 & 1 & - \\
\hline \multirow{2}{*}{ Educational status } & Illiterate & 16 & 395 & 1 & - \\
\hline & Literate & 305 & 1229 & $6.1(3.6-10.257)$ & ** \\
\hline \multirow{2}{*}{ Parity } & Nuilpara & 39 & 62 & 1 & - \\
\hline & Multiypara & 282 & 1562 & $3.4(2.28-5.304)$ & ** \\
\hline \multirow{2}{*}{ Contraceptive user } & Yes & 274 & 1213 & $1.9(1.421-2.7)$ & ** \\
\hline & No & 47 & 411 & 1 & \\
\hline \multirow{2}{*}{ STI history } & Yes & 213 & 255 & $10.5(8.10-13.8)$ & $8.3(5.6-12)^{*}$ \\
\hline & No & 108 & 1369 & 1 & 1 \\
\hline \multirow{2}{*}{ HIV status } & positive & 39 & 26 & $8.5(5.0-14.1)$ & $9.0(4.5-18)^{*}$ \\
\hline & Negative & 282 & 1598 & 1 & 1 \\
\hline \multirow{2}{*}{ Age at first intercourse } & $<18$ & 270 & 950 & $3.7(2.741-5.1)$ & $8.9(5.5-14)^{\star}$ \\
\hline & $\geq 18$ & 51 & 674 & 1 & 1 \\
\hline \multirow{2}{*}{ MSP } & Yes & 162 & 48 & $33.4(23-47.9)$ & $40(22.8-70)^{*}$ \\
\hline & No & 159 & 1576 & - & - \\
\hline \multirow{2}{*}{ Cigarette smoking } & Yes & 30 & 65 & $2.4(1.5-3.87)$ & ** \\
\hline & No & 291 & 1559 & 1 & - \\
\hline
\end{tabular}

Table 3: Bivaraite and multivariate analysis of risk factors associated with cervical cancer at Yirgalem General Hospital. 
Citation: Hailemariam T, Yohannes B, Aschenaki H, Mamaye E, Orkaido G, et al. (2017) Prevalence of Cervical Cancer and Associated Risk Factors among Women Attending Cervical Cancer Screening and Diagnosis Center at Yirgalem General Hospital, Southern Ethiopia. J Cancer Sci Ther 9: 730-735. doi:10.4172/1948-5956.1000500

with (COR=3.756 :95\% CL: 2.741,5.147). Multiple sexual partners were 33.453 times having risk to develop cervical cancer than those who had no multiple sexual partners (COR=33.453: 95\% CI: 23.315, 47.998). According to cigarette smoking status, those smokers were 2.473 times having risk to develop cervical cancer than those who have no cigarette smoking exposure (COR=2.473: 95\%: CI 1.576, 3.879). Women who had suffered with any sexually transmitted disease were 10.588 times more likely to develop cervical cancer as compared to those women who had never suffered any sexually transmitted disease $(\mathrm{COR}=10.588$ : $95 \%$ CI :8.104,13.83). The study reveals that, being positive with HIV were 8.5 times risk to develop cervical cancer than those women who have no HIV positive (COR=8.5: 95\% CI: 5.093,14.185). Women those who reported to have more than one sexual partners in the past had 33 times increased odds of having cervical cancer as compared to women those who had a single casual partner $(\mathrm{COR}=33: 95 \% \mathrm{CI}$ : 23.315,47.998). The odds of developing cervical cancer was 2.47 times more among ever smokers as compared to never smokers $(\mathrm{COR}=2.47$ : 95\% CI:1.576,3.879). Variables became significant on bivariate analyses or $\mathrm{p}$-value $<0.25$ were re-entered in to Multivariate analysis to control for possible potential confounders. Risk factor investigated for their causal were maternal education, address of the mother, occupation status, marital status, parity, gravidity, number of sexual partners, contraceptive use, HIV status, STI history, age at first inter course and cigarette smoking. Of the total variables, only, being HIV positive, having STI history, age at first inter course, and having multiple sexual partner were found to be independently and significantly associated with the likelihood of having cervical cancer while the rest variables were no significant association during multivariate analysis after controlling for potential confounders in fact they became significant during multivariate analysis.

\section{Discussion}

This study reveals that, 7 years cervical cancer prevalence is $16.5 \%$. Urban resident, literacy, occupational status employed, number of delivery increased, HIV status positive, having multiple sexual partners, history of STI exposure, using contraceptives, exposure of cigarette smoking and initiation of early sexual intercourse are variables associated in bivariate analysis. After controlling for possible potential confounders, HIV positive, multiple sexual partners, having STI, and early sexual intercourse independently and significantly associated with cervical cancer. Having multiple sexual partners increases the chance of acquiring. Studies that have been conducted in both rural and urban areas show that significant association between having multiple sexual partners and cervical cancer [11]. A study in Morocco shows that promiscuity and polygamist which is related to multiple sexual partners increases the risk of cervical cancer [11]. The finding of this study reveals that multiple sexual partners are significantly associated with cervical cancer as compared to those women who have no exposure of multiple sexual partners ( $\mathrm{AOR}=40 ; 95 \% \mathrm{CI}: 22.44,70.204)$ and having history of STI exposure and being HIV positive became significant with the outcome variable of the study as compared to those women who have no history of STI exposure and HIV negative (AOR=8.3:95\%CI: 5.639,12.405) and (AOR=9:95\% CI: 9:4.537,17.985) respectively. This is similar with the study that has been conducted on cervical cancer screening program in Beijing [32]. A study in Zambia among HIV infected women states that HIV positive women have higher likelihood of having high grade cervical squamous intra epithelial lesion $(\mathrm{AOR}=8.0$ :95\% CI: 20.54,52.04) and cervical cancer cases are reported to occur in more in the immune suppressed cases [4]. Early sexual debut is reported more documented among cervical cancer patients than the late counterparts. This is mainly because at young age the cervix has immature membrane thus making it susceptible to oncogenic agent particularly (HR HPV) Cooper) [24,25]. In this study, women who began first intercourse at early age became significant with cervical cancer as compared to those who did not begin first intercourse at early age (AOR=8.9:95\%CI: $5.588,14.393$ ). The same type of finding was consistently reported that initiation of sexual practice too early became a risk factor in most previous epidemiological studies of cervical cancer $(\mathrm{AOR}=8.9: 95 \%$ CI $8.38,11.983)[34-40]$.

\section{Conclusion}

From the screened and diagnosed clients in consecutive 7 years, the prevalence of the cervical cancer is $16.5 \%$. The risk factors that became significant with CC are maternal occupation, education status, mothers address, having multiple sexual partners, being HIV positive, exposure of STI and early age at initiation of sexual intercourse are identified predictors of the cervical cancer as compared to non-cancerous patients by using bivariate analysis. By using multivariate analysis after controlling the possible potential confounders, being HIV positive, having multiple sexual partners, having STI history, and initiation of early sexual intercourse became independently and significantly associated with cervical cancer.

1. Strengthening both cancer prevention control programs and implementation strategies by addressing socio-demographic, behavioral and reproductive factors of the study became very crucial.

2. Giving sexuality education tailored to age and culture, early screening and treatment for cervical cancer and provision of condoms for those who have practice of having multiple sexual partners, STI including HIV.

3. Advocate delaying of age at initiation of sexual intercourse too early to mitigate the preventive and controllable cause of cervical cancer.

4. Prepare consistent mass campaign for cervical cancer screening and diagnosis as it is vital to track the cases early.

5. For researchers: Comprehensive and qualitative study became recommended as this study was limited to assess the prevalence and associated risk factors of cervical cancer at Yirgalem general hospital.

6. Responsibility should be taken by government, nongovernmental organizations, and other collaborative stakeholders so as prevent the predictors of this study; these are having multiple sexual partners, being HIV positive, exposure of STI and early age at initiation of sexual intercourse.

\section{Competing Interests}

The authors declare that they have no competing interests in this section.

\section{Acknowledgment}

We would like to express our deep appreciation and sincere thanks to Hawasa College of health science for its giving this chance and financial support during the research work. We would like to extend our thanks to data collectors and yirgalem hospital administration for helping us by giving relevant information during the research work.

\section{References}

1. Getachew E (2015) Knowledge attitude and practice on cervical cancer and screening among reproductive health service clients, Addis Ababa, Ethiopia. 1: $1-6$ 
Citation: Hailemariam T, Yohannes B, Aschenaki H, Mamaye E, Orkaido G, et al. (2017) Prevalence of Cervical Cancer and Associated Risk Factors among Women Attending Cervical Cancer Screening and Diagnosis Center at Yirgalem General Hospital, Southern Ethiopia. J Cancer Sci Ther 9: 730-735. doi:10.4172/1948-5956.1000500

2. Ndoh G (2010) National Department of Health Strategic Plan 2010/11-2012/13 Department of Health, Republic of South Africa.

3. Hacer GS, Lale T, Wright TC Jr (2011) Determining nurse-midwives' knowledge of the PapSmear test and their rate of being tested in Turkey. Asian Pac J Cancer Preven 2: 17

4. Gopal K (2012) Singh disclosures j community health, rural-urban trends and patterns in cervical cancer mortality, incidence, stage, and survival in the United States. 37: 217-223

5. WHO/ICO (2014) Information centre on HPV and cervical cancer (HPV Information Centre), author Summary report on HPV and cervical cancer statistics in Ethiopia, Ethiopia.

6. Echelman D, Feldman S (2012) Management of cervical precancers: Global perspectives. Hematol Oncol Clin N Am 26: 32-44.

7. Yesuf T (2012) Survival and associated factors among cervical cancer patients in Black Lion Hospital, Addis Ababa, Ethiopia.

8. Department of Health (2000) Pretoria. National guideline for cervical cancer screening program. South Africa.

9. The GLOBOCAN database (2012) Cancer incidence, mortality and prevalence worldwide. International Agency for Research on Cancer. Lyon, France.

10. Getahun F, Mazengia F, Abuhay M, Birhanu Z (2013) Comprehensive knowledge about cervical cancer is low among women in Northwest Ethiopia. BMC Cancer 14: 2

11. Obure J, Olola O, Swai B, Mlay P, Masenga G, et al. (2009) Prevalence and severity of cervical squamous intraepithelial lesion in a tertiary hospital in northern Tanzania. Tanzan J Health Res 11:163-69.

12. Hawes SE, Critchlow CW, Niang MAF, Diouf MB, Diop A, et al. (2003) Increased risk of high-grade cervical squamous intraepithelial lesions and invasive cervical cancer among African women with human immunodeficiency virus type 1 and 2 infections. J Infect Dis 555-563.

13. WHO (2013) International agency for research on cancer press release. Lyon, France. p. 223

14. Aklimunnessa K (2006) Effectiveness of cervical cancer screening over cervical cancer mortality among Japanese women. Jpn J Clin Oncol 36: 511-518.

15. https://bmccancer.biomedcentral.com/articles/10.1186/1471-2407-13-2

16. http://conference.ncri.org.uk/abstracts/2014/abstracts/B151.htm/

17. United Nations Population Fund Agency (UNFPA) (2011) Comprehensive cervical cancer prevention and control program guidance for countries.

18. Pathfinder International (2010) Combating cervical cancer in Ethiopia. Addis Tesfa project, Ethiopia.

19. Carey P, Gjerdingen, J Fam Prac DK (1993) Follow-up of abnormal Papanicolaou smears among women of different races 37: 583-87.

20. Rweyemamu KY (2013) factors associated with cervical cancer among women attending referral hospitals in dar es salaam, Tanzania. Muhimbili University of Health and Allied Sciences, Tanzania.

21. Meinhardt G, Gissmannl L, Pawlita M (1992) Human Papillomavirus (HPV) infection, HIV Infection and Cervical Cancer in Tanzania, East Africa. Int J Cancer 521: 515-21.

22. Sitas F, Madhoo J, Wessie J (2000) Incidence of histologically diagnosed cancer in South Africa 1993-1995. Johannesburg National Cancer Registry of South Africa. South African Institute of Medical Research

23. Ocean Road Cancer Institute (2008) Annual Report 2008. Dar es Salaam, Tanzania.

24. Alarco C, Tera M, Herna D (2009) Cervical carcinoma in Southern Mexico: Human papillomavirus and cofactors 32: 300-307.

25. Herrero R, Meijer CJ, Shah K, Franceschi S, Louie KS, et al. (2009) Early age at first sexual intercourse and early pregnancy are risk factors for cervical cancer in developing countries. Br J Cancer 1191-1197.

26. International Agency for Research on Cancer (2008) World Health Organization GLOBOCAN. Geneva, Switzerland.

27. Massad LS, Seaberg EC, Watts DH, Hessol NA, Melnick S, et al. (2004) Low incidence of invasive cervical cancer among HIV-infected US women in a prevention program. AIDS 18: 109-113.

28. Newton R, Ziegler J, Casabonne D, Beral V, Mbidde E, et al. (2007) A case-control study of cancer of the uterine cervix in Uganda. Euro $\mathrm{J}$ Cancer Prevention16: 555-558.

29. Bayo S, Bosch FX, Sanjosé S De, Muñoz N, Combita L, et al. (2002) Risk factors of invasive cervical cancer in Mali. Int J Epidemiol 31: 2029

30. Hammouda D, Mun N, Herrero R, Arslan A, Bouhadef A, et al. (2005) Cervical carcinoma in Algiers, Algeria: Human papillomavirus and lifestyle risk factors. Int J Cancer pp. 483-489.

31. Reis N, Beji N, Kilic D (2011) Risk factors for cervical cancer: Results from a hospital-based case-control study. Int J Hematol Oncol 153-159.

32. Tao L, Han L, Li X, Gao Q, Pan L, et al. (2014) Prevalence and risk factors for cervical neoplasia: A cervical cancer screening program in Beijing. BMC Public Health 483-523.

33. Zewdie MD, Fessahaye AT, Henok AF (2015) Prevalence and factors associated with VIA positive result among clients screened at family guidance association of Ethiopia, south west area office, Jimma model clinic, Jimma, Ethiopia 2013: A cross-sectional study. BMC Res Notes 8: 618.

34. Forouzanfar MH, Foreman KJ, Delossantos AM, Lozano R, Lopez AD, et al (2011) Breast and cervical cancer in 187 countries between 1980and 2010: a systematic Analysis. Lancet 378: 1461-1484

35. World Health Organization (2002) Cervical cancer screening in developing countries: Report of WHO consultation. France.

36. Federal Democratic Republic of Ethiopia Ministry of Health (2015) Guideline for cervical cancer prevention and control. Ethiopia.

37. http://jjco.oxfordjournals.org/ at Addis Ababa University Libraries on July 25, 2014.

38. Terefe Y, Gaym A (2008) Knowledge, attitude and practice of screening for carcinoma of the cervix among reproductive health clients at three teaching hospitals. Ethiopian J Reproduc Health.

39. Eze JN, Umeora OU, Obuna JA, Egwuatu VE, Ejikeme BN (2012) Cervical cancer awareness and cervical screening uptake at the Mater Misericordiae Hospital, Afikpo, Southeast Nigeria. Ann Afr Med 11: 238-243

40. Getachew T (2004) A study of knowledge, attitude and practice of screening procedure for carcinoma of the cervix among nurses in Addis Ababa. Int $J$ Womens Health 9: 365-372. 